\title{
Early warning signals of tipping points in periodically forced systems
}

\author{
Mark S. Williamson ${ }^{1}$, Sebastian Bathiany ${ }^{2}$, and Timothy M. Lenton ${ }^{1}$ \\ ${ }^{1}$ Earth System Science group, College of Life and Environmental Sciences, University of Exeter, \\ Laver Building, North Park Road, Exeter EX4 4QE, UK \\ ${ }^{2}$ Aquatic Ecology and Water Quality Management, Wageningen University, PO Box 47, \\ Wageningen, the Netherlands
}

Correspondence to: Mark S. Williamson (m.s.williamson@exeter.ac.uk)

Received: 27 October 2015 - Published in Earth Syst. Dynam. Discuss.: 6 November 2015

Revised: 4 February 2016 - Accepted: 26 March 2016 - Published: 13 April 2016

\begin{abstract}
The prospect of finding generic early warning signals of an approaching tipping point in a complex system has generated much interest recently. Existing methods are predicated on a separation of timescales between the system studied and its forcing. However, many systems, including several candidate tipping elements in the climate system, are forced periodically at a timescale comparable to their internal dynamics. Here we use alternative early warning signals of tipping points due to local bifurcations in systems subjected to periodic forcing whose timescale is similar to the period of the forcing. These systems are not in, or close to, a fixed point. Instead their steady state is described by a periodic attractor. For these systems, phase lag and amplification of the system response can provide early warning signals, based on a linear dynamics approximation. Furthermore, the Fourier spectrum of the system's time series reveals harmonics of the forcing period in the system response whose amplitude is related to how nonlinear the system's response is becoming with nonlinear effects becoming more prominent closer to a bifurcation. We apply these indicators as well as a return map analysis to a simple conceptual system and satellite observations of Arctic sea ice area, the latter conjectured to have a bifurcation type tipping point. We find no detectable signal of the Arctic sea ice approaching a local bifurcation.
\end{abstract}

\section{Introduction}

The potential for early warning of an approaching abrupt change or "tipping point" in a complex, dynamical system has been the focus of much research, see for example Wiesenfeld (1985), Held and Kleinen (2004), Thompson and Sieber (2011) and Scheffer et al. (2012). Abrupt change in a system can occur due to a bifurcation - that is, a small smooth change in parameter values can result in a sudden or topological change in the system's attractors. This extreme sensitivity of systems close to criticality is familiar from studies of critical phenomena in statistical mechanics (Domb et al., 1972-2001) and stability analysis in nonlinear dynamical systems (Kuznetsov, 2004). Much work on the anticipation of bifurcations from time series data, e.g. in ecosystems (Carpenter et al., 2011), or the climate system (Dakos et al., 2008; Lenton, 2011), is based on methods that infer the time taken for the system to recover to its steady state (the system's timescale) from perturbations away from that steady state. The methods are usually based on a clear separation of three timescales: (i) The timescale of the dynamics of the system one wants to study, (ii) much faster processes than the timescale of the system, usually thought of as the perturbations the system recovers from and (iii) much slower processes than the timescale of the system which govern the present system steady state. In addition, the system dynamics are modelled as overdamped, the fast dynamics as a noisy, normally distributed random variable of small variance and the slow dynamics as a constant, control parameter. Provided these are good working approximations, critical slowing down - the increase of the system's timescale, is expected prior to a local bifurcation and can be detected by computing the lag 1 autocorrelation in a sliding window of a system's 
time series. An increasing trend in autocorrelation shows the stability of the system is weakening or equivalently, the system's timescale is increasing - which is a generic feature of a system approaching a local bifurcation. Provided the variance of the fast noisy process is constant, increasing variance of the system's time series is also a good indicator of critical slowing down, although it is less robust than lag 1 autocorrelation due to its dependence on the noisy process.

For many systems of interest one or more of the above assumptions may be invalid (Williamson and Lenton, 2015). In particular, when the forcing of a system has a comparable period to the timescale of the system, the forcing cannot be modelled as a slow, constant control parameter or a fast, random process; however they can still be thought of as a perturbation away from the system steady state that one can measure the recovery time from, an observation we exploit in this manuscript. These systems are particularly relevant in the climate system where periodic forcing is a consequence of the motion of the Earth relative to the Sun; for example, solar insolation variation from the diurnal, annual or Milankovich cycles. These systems have steady states described by periodic attractors rather than the simpler, fixed point type attractors required for lag 1 autocorrelation and variance to be used as early warning indicators.

In an elegant study Wiesenfeld (1985) computed the Fourier spectra of noisy perturbations in systems with periodic attractors. Very close to a local bifurcation, the dominant system timescale asymptotes towards infinity causing the dynamics of the noisy perturbations away from the attractor to be dependent only on the type of bifurcation and not on the details of the system's specific equations. This observation allowed the author to classify all codimension 1 bifurcations in an arbitrary periodic system by the harmonics in the spectra of residuals. He called these early warning signals noisy precursors.

A common method to study stability changes in periodic attractors is the return or Poincaré map, see Strogatz (2001). Here, one converts the continuous-time periodic orbit into the fixed point of a return map by sampling the orbit once every period. One can then compute the usual fixed point indicators for the resulting return map time series such as lag 1 autocorrelation and variance. Advantages of this approach include no linearity requirement on the dynamics of the periodic attractor although perturbations away from the attractor must be small enough to be treated linearly. One can also handle systems with internally generated cycles rather than those generated by external periodic forcing that we look at in this manuscript. To detect any change in stability from the return map, the timescale of the system must also be greater than the period of the forcing. To see this, imagine one samples the cycle to create a point in the return map and then immediately after perturbs the system away from the stable cycle. If the system timescale is shorter than the cycle period, which is determined by the forcing period, the system will have recovered back to the stable cycle before the sys- tem is sampled again for the next point in the return map. The perturbation and its recovery will therefore be invisible to stability analysis on the return map time series. It should be noted that close to a local bifurcation the system timescale approaches infinity so satisfying this requirement. However, if this requirement is met only over a few cycles or less it will be very hard to detect.

With this limitation in mind we suggest alternative early warning signals of approaching local bifurcations when the period of the forcing is similar to the timescale of the system. We look particularly at sinusoidal forcing since this approximates the variation of solar insolation well. However, the method works for any periodic forcing and we give the derivation of the general case in the Appendix. We demonstrate that increasing the system timescale as it approaches a local bifurcation shows up as an increasing phase lag in the system response relative to the forcing. In addition, the amplitude of the system response increases as well. These indicators, like lag 1 autocorrelation and variance in fixed point attractor methods, assume the linearised dynamics approximate the true nonlinear dynamics well. One might ask how well the linear approximation works, especially near the bifurcation, since bifurcations are strictly nonlinear phenomena. A quantitative answer to this question can be provided by computing the Fourier spectrum of the system's time series. In particular, as the system's behaviour becomes more nonlinear, harmonics of the forcing period are generated in the system response and their amplitudes may be obtained from the system's Fourier spectra. Since the system response becomes more nonlinear as one approaches the bifurcation, one can view the increasing amplitude of harmonics as another early warning signal.

The paper is organised as follows: in Sect. 2 the early warning indicators used in the manuscript are introduced, namely the system response phase lag and amplification as well as harmonic amplitudes. We also review a common approach to periodic attractors, the return map, which is complementary to phase lag and response amplification. In Sect. 3 a periodically forced overdamped system in a double well potential is used to illustrate the timescale separation problem and the properties of the early warning indicators when a local bifurcation is approached. In Sect. 4 we apply the early warning indicators to satellite observations of Arctic sea ice area, a system conjectured to be approaching a local bifurcation. We conclude in Sect. 5 .

\section{Early warning indicators of local bifurcations in periodic systems}

As previously mentioned in the introduction, the Arctic seaice has been conjectured to be approaching a local bifurcation. Treating this system approximately, one can think crudely of the slow control parameter as the decrease of outgoing long-wave radiation, giving a warming trend in air 
temperature as the Earth's atmospheric $\mathrm{CO}_{2}$ concentration increases. This is a system that is forced periodically and deterministically by the annual cycle of short-wave solar insolation. The system response is dominated by this periodic forcing rather than small amplitude; random noisy forcing is also present and system timescale is roughly the same order of the forcing period. In this section, motivated by detection of local bifurcations in systems like the sea-ice type from time series, we look for suitable methods. Although this system has no clear separation of timescales with which to use fixed point methods directly, the fact that this system has a large and predictable perturbation one can measure the response to reduces the need for the statistical methods (and therefore large numbers of data) required for noisy perturbations and so the number of data in a time series becomes less of an issue.

Students of physics or engineering will likely have solved the equation for the forced damped harmonic oscillator and observed in the overdamped limit that the phase and amplitude depend on the damping parameter (see for example Main, 1993). In the following subsections we propose to use this fact and phase lag and response amplification as simple non-statistical indicators of system timescale. We demonstrate their properties and their functional dependence on system timescale. These early warnings are based on a linear dynamics approximation but by taking the Fourier transform of the system response, one can also look at the magnitude of the nonlinear response. This has two purposes, first one can check the linear approximation is good and second, because bifurcations are strictly nonlinear phenomena, the system response will become more nonlinear as one approaches the bifurcation giving another early warning indicator that can be monitored.

The systems we concentrate on in this manuscript, relevant to externally forced climate problems, have cycle periods determined by the period of forcing and a one-way coupling from the forcing to the system and so are special cases of periodic attractors. For these special cases, when forcing period and system timescale are similar, phase lag and response amplification are useful indicators. However, return maps are generally more useful when treating more general periodic attractors. At the end of the section we briefly review the method of return maps.

\subsection{Phase lag and response amplification}

We consider systems that can be described by

$\dot{x}=f(x)+D(t)$

where $f(x)$ is, generally, a nonlinear function of the system state scalar variable $x$ with forcing $D(t)$ given by

$D(t)=D_{m}+D_{a} \cos (\omega t)$.

$D_{m}$ and $D_{a}$ are constants, $\omega=\frac{2 \pi}{T}$ is the angular frequency and $T$ is the period of the forcing. We have assumed any other random, noisy external forcing to be very small and can be neglected. The solution for a general form for $D(t)$ is given in the Appendix, however here we use sinusoidal forcing as this is most relevant for many climate systems and we wish not to obscure the simplicity of the main result. $\dot{x}$ describes the dynamics of a forced overdamped system. This is a nonautonomous system whose state can be completely described by $t$ and $x$. After some time $t_{s} \gg \tau$, where $\tau$ is the system timescale, the system will settle into some sort of steady state, either an orbit or a fixed point whose mean state $\bar{x}$ is

$\bar{x}=\frac{1}{T} \int_{t_{s}}^{T+t_{s}} x(t) \mathrm{d} t$.

We now Taylor expand $f(x)$ to first order around $\bar{x}$ so that

$\dot{x} \approx a-\frac{x}{\tau}+D_{a} \cos (\omega t)$,

where

$a=f(\bar{x})-\left.\frac{\partial f}{\partial x}\right|_{x=\bar{x}} \bar{x}+D_{m}$

$\tau=-1 /\left.\frac{\partial f}{\partial x}\right|_{x=\bar{x}}$

are the linearisation constants. We have assumed higher order terms such as $\frac{1}{n !} \frac{\partial^{n} f}{\partial x^{n}}(x-\bar{x})^{n}, n \geq 2$ are small relative to zeroth and first order terms so that the linearised dynamics approximates the full nonlinear dynamics well. We show how to check this approximation in Sect. 2.2. Assuming the approximation is good, one can solve Eq. (4). As $t \gg \tau$ the system settles into the orbit

$\lim _{t \gg \tau} x(t)=a \tau+\frac{D_{a} \tau}{\sqrt{1+\omega^{2} \tau^{2}}} \cos (\omega t+\phi)$,

where the system response lags the forcing by phase $\phi_{\text {lag }}=$ $\omega t_{\text {lag }}=-\phi$ given by

$\phi_{\text {lag }}=\arctan (\omega \tau)$,

that is, the phase lag is a function of the forcing frequency and the system response timescale. One also notices that the system response, relative to the forcing amplitude, $D_{a}$, is amplified by a factor

$\frac{\tau}{\sqrt{1+\omega^{2} \tau^{2}}}$,

which is also a direct function of $\omega$ and $\tau$. The more general derivation when $D(t)$ can be any periodic function is given in the Appendix Sect. A.

\subsection{System nonlinearity and harmonic amplitude from Fourier analysis}

By simply looking at the time series of the system response and the forcing one can determine what the amplitude and 
phase lag are when the driving is of the form Eq. (2) and the system response is approximately linear without the need for statistics. However, the system is essentially nonlinear and these nonlinear effects may become large near a bifurcation or when the system is driven hard. By taking the Fourier transform of the time series of the system response one can quantify how large these nonlinear effects are. With a similar motivation Wiesenfeld (1985) and Wiesenfeld and McNamara (1986) calculated the Fourier spectra of the perturbations, rather than the response, away from periodic attractors very close to local bifurcations with noisy and weak periodic modulation respectively.

Once the system has settled into an orbit of period $T$, the full nonlinear response of an arbitrary system can be written as a Fourier series, a sum of $N$ sinusoidal functions with angular frequencies $\omega_{n}=\frac{2 \pi n}{T}$, amplitudes $A_{n}$ and phases $\phi_{n}$, i.e.

$x(t)=\sum_{n=0}^{N} A_{n} \cos \left(\omega_{n} t+\phi_{n}\right)$

The $n=0$ component is a constant, the long-term mean of the response; the $n=1$ component is the linear response of the system and the $n \geq 2$ components are the $n$th order harmonics and come about from the nonlinear response of the system. Since the system has settled into a periodic orbit the system must repeat itself every cycle. The only way the system can do this is by adding harmonics to linear response. By looking at the ratios $A_{n} / A_{1}$ for $n \geq 2$ the nonlinear effects relative to the linear approximation can be quantified. In practice the largest harmonics will generally be the 2nd $(n=2)$ and 3rd order $(n=3)$ harmonics and provided they are an order of magnitude (10 times, $A_{n} / A_{1}<10^{-1}$ ) less than the fundamental harmonic, the linear analysis in the last section works well. Calculation of the amplitudes, $A_{n}$, can be made via a Fourier transform of the time series.

One may also expect subharmonics, components that have periods that are integer multiples of the forcing period, to be observed in the system response. Subharmonics are not possible in the systems we consider here due to the dimensionality of the phase space. ${ }^{1}$

Since the ratios $A_{n} / A_{1}$ measure how nonlinear the system is one expects these to increase as the system approaches a

\footnotetext{
${ }^{1}$ Systems described by Eq. (1) are completely described by the two-dimensional space of variables $x$ and $t$. Recasting the nonautonomous system in Eq. (1) as a two dimensional autonomous system by identifying a new angular variable $\phi=\omega t$, the system is then described by $\dot{x}=f(x)+D(\phi)$ and $\dot{\phi}=\omega$. The resulting phase space $(x, \phi)$ is then cylindrical as $\phi$ is $2 \pi$ modular. If subharmonics are possible in the periodic system response the trajectory must wind around the cylinder at least twice before repeating itself. Such a trajectory implies it crosses itself which is not allowed due to the existence and uniqueness theorem. Therefore subharmonics cannot exist in the two-dimensional systems. This is of course not true for three- and higher-dimensional systems.
}

bifurcation. These ratios can be plotted for a time series by taking the Fourier transform for a data window consisting of an integer number of cycles and sliding this window forward by one cycle recursively through the time series. The number of cycles in the window must be large enough that the harmonics can be satisfactorily resolved in the Fourier spectra. In addition, each cycle must be sampled at a time interval $\Delta t \leq T_{\text {Nyquist }} / 2$ where $T_{\text {Nyquist }}$ is the minimum harmonic period you want to resolve.

\subsection{Lag 1 autocorrelation of a return map}

Provided the system timescale is larger than its period, $\tau / T>1$, one can use return maps to assess the stability of a periodic attractor. The return map time series, generated by sampling the system response once every cycle, allows one to apply fixed point statistical early warning indicators such as lag 1 autocorrelation. It is the random forcing away from the periodic attractor that this method infers timescale from, rather than response to deterministic, periodic forcing. This is usually done by calculating the lag 1 autocorrelation for a sliding data window of the return map time series at least as long as the system timescale but not so long that any increasing trend in system timescale skews the autocorrelation estimate. It is also desirable to have many points within this window as the standard error of the estimate scales as $1 / \sqrt{m}$, where $m$ is the number of cycles (points) within the window (see Williamson and Lenton (2015) for a discussion). For time series consisting of a small number of cycles this can be a limiting factor.

\section{Examples}

We now demonstrate the early warning indicators in Sect. 2 for different ratios of forcing period, $T$, (or equivalently angular frequency $\omega$ ) to system timescale $\tau$. In particular, we use a periodically forced double well potential as our main system. This system has been extensively studied in the context of stochastic resonance (see McNamara and Wiesenfeld (1989) and Gammaitoni et al. (1998) for reviews) as the simplest model of the phenomena when noise is also added. Phase and amplitude have been investigated in this setting by Shneidman et al. (1994) and Jung and Hänggi (1993). This literature is largely concerned with resonance effects in transition probabilities between the wells (finite barrier height between the wells) rather than the anticipation of local bifurcations (barrier height tends to zero).

Our system which has one dynamical variable, $x$, and evolves according to

$\dot{x}=x-x^{3}+D(t)$,

where overdots denote differentiation with respect to time, $t$ and the periodic forcing function $D(t)$ is given by Eq. (2). Equation (11) models a nonautonomous nonlinear system, 
the overdamped limit of a Duffing oscillator (Thompson and Stewart, 2002). When forcing is constant $(\omega=0)$ the familiar, well-studied autonomous fold bifurcation is recovered (for example see Strogatz, 2001). For $\omega=0$, the solutions of $\dot{x}=0$, give the system's fixed points, $x^{*}$ (the nullclines) and number either one or three depending on the value of $D_{m}$. One can evaluate the stability of these fixed points by looking at the linearised dynamics close to the fixed points, $J\left(x^{*}\right)$ :

$J\left(x^{*}\right)=\left.\frac{\partial \dot{x}}{\partial x}\right|_{x=x^{*}}=1-3 x^{* 2}$.

If $J\left(x^{*}\right)$ is negative, the fixed point is stable, if it is positive it is unstable. In the region where three fixed points exist one finds a bistable region, i.e. two points are stable while the third is unstable. The bistable region has boundaries marked by the local bifurcations and these can be found by solving $J\left(x^{*}\right)=0$ for $x^{*}$ when the fixed point becomes neutrally stable. One can also calculate the $e$-folding timescale of the system in state $x, \tau$ from the Jacobian $\tau=-1 / J(x)$. We will refer to the $e$-folding time as the system timescale. As the system gets closer to the bifurcation the system timescale will increase and tend to infinity at the bifurcation. Early warning indicators are simply functions of $J\left(x^{*}\right)$ or equivalently $\tau$.

\subsection{Period of forcing similar to system timescale, $\omega \tau \sim 1$}

This regime, $T \sim \tau$, is the main focus study in this manuscript, when the system responds on approximately the same timescale as the period of the forcing. In this regime the dynamics are a balance between the system's tendency to want to decay towards the fixed point and the forcing trying to push it away. After some time, $t \gg \tau$, the system will settle into an orbit rather than a fixed point due to the similarity of the timescales. Just as there was a bistable region where multiple stable fixed points existed for a single value of $D_{m}$ when $\omega=0$, analogously in the case $T \sim \tau$ multiple stable periodic attractors are possible given a fixed set of values for $D_{m}, D_{a}$ and $\omega$. The system state $x$ is plotted against $D$ and against $t$ as the blue line in Fig. 1 . Which state the system settles in depends only on the system's initial condition $x(t=0)$. Local bifurcations are present in this intermediate region, however they are local bifurcations between orbits rather than fixed points. In this intermediate regime, one can neither place the $D_{a} \cos (\omega t)$ part of $D(t)$ in either the slow or fast processes and therefore the assumptions of the usual fixed point early warning methods are not strictly valid. This is, however, where phase lag and response amplification are useful early warning indicators.

To illustrate the early warning indicators we fix the forcing amplitude $D_{a}$ and the period $T \sim O(\tau)$ and take $D_{m}$ as a control parameter, slowly varying from negative values towards the local bifurcation in the system described by Eq. (11). We expect to see the system response become more phase lagged and amplified as we approach the local bifur-

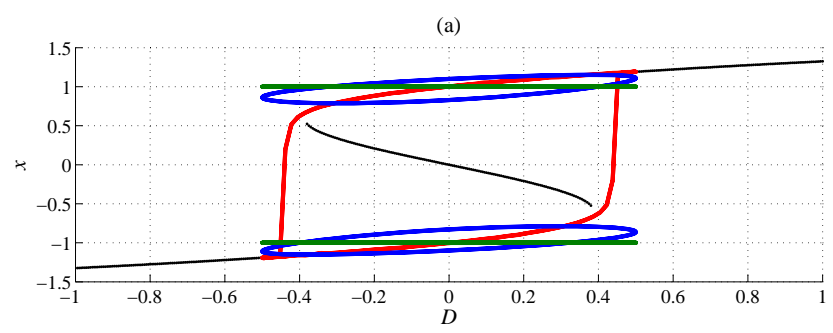

(b)

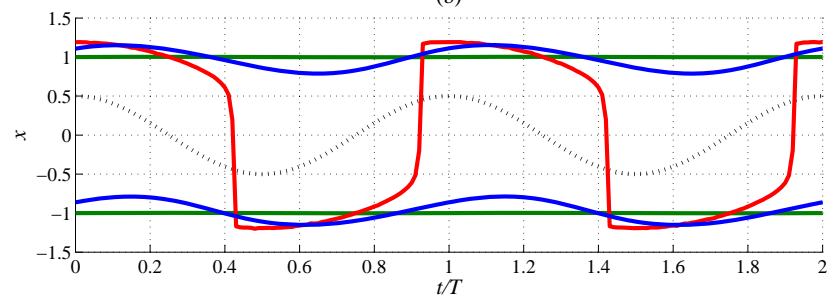

Figure 1. The dynamics of the system described by Eq. (11) in three different timescale regimes. Forcing parameters are set to $D_{m}=0$, $D_{a}=1 / 2$. In the upper panel system state $x$ is plotted against $D(t)$. The black lines are the nullclines and the coloured lines are the system responses for different periods of forcing. In the lower panel $x$ is plotted against the number of cycles, $t / T$, once the system has reached a steady state. The dotted line is the forcing, $D(t)$ while the coloured lines are the system responses. The red line is for the slow forcing limit, $\tau \ll T, T=100 \pi$ so $\omega \tau \approx 1 / 100$. As the system timescale is much faster than the change in the forcing, the system essentially "sticks" to the fixed points until they become unstable at the bifurcations and jump to a different attractor. One can regard the system response in two different ways: (i) a single periodic attractor giving relaxation oscillations in a monostable region. (ii) Tipping between point attractors by crossing local bifurcations in a bistable region. This tipping causes the dynamics to be very nonlinear. The green line is the fast forcing limit, $T \ll \tau$, $T=\pi / 100$ so $\omega \tau \approx 100$. There are two possible stable attractors for this set of values. As the system timescale is much slower than the change in the forcing, the system essentially remains static and all the dynamics come from the forcing itself. Although it is hard to see in the figure due to the small amplitude system response, the lag relative to the forcing is $1 / 4$ of a cycle and the dynamics are approximately linear. The blue line is the intermediate regime, $\tau \sim T$, $T=\pi$ so $\omega \tau \approx 1$ and there are two possible stable attractors for this set of values. As the system timescale is approximately the same as the period of the forcing, the system response is a competition between the system's tendency to decay towards the nullcline and the forcing pushing it away setting up a stable orbit. Notice that there is some phase lag and the dynamics look approximately linear.

cation at $D_{m} \approx 0.33$ when approaching from the lower nullcline solutions. We also expect the amplitude of the harmonics of the system response to increase.

We choose to tip the system from one state to another by slowly altering the mean of the driving $D_{m}$. Alternatively, the system could have tipped by changing one of the other driving parameters such as amplitude $D_{a}$ or frequency $\omega$. Since the system response amplitude depends on $D_{a}$ and $\omega$ and 
(a)

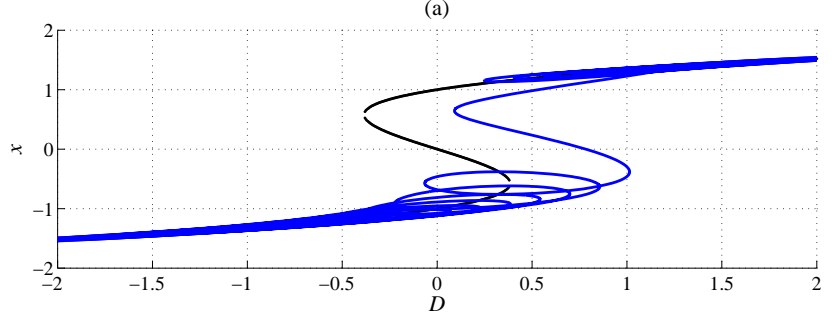

(b)

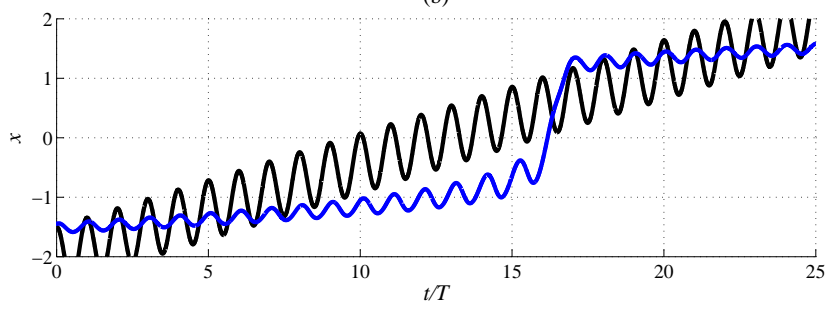

Figure 2. The dynamics of the system are described by Eq. (11) with varying $D_{m}$. Parameters are set to $D_{a}=1 / 2, T=\pi$ (the same order as the system timescale $\omega \tau \sim 1$ ) and $D_{m}$ is varied linearly with time between -2 and 2 over about 25 cycles. In the upper panel the black lines are the nullclines while the system response is the blue line plotted against $D(t)$. The orbit loses stability around a mean value of $D \approx 0.5$ and jumps to a new orbit. In the lower panel we have plotted the system response (blue) against the forcing $D$ against $t / T$. One can see the loss of stability of the orbit around $t / T \approx 15$ and the prior increase in system response amplitude.

phase lag depends on $\omega$, one must take this into account when inferring system timescales from the indicators.

In Fig. 2 the system is run forward in time, linearly varying $D_{m}$ from -2 to 2 across the bifurcation over about 25 cycles of the forcing period (for the values of the parameters see the figure caption). Plotted in Fig. 3 are phase lag and amplitude of the system response prior to the bifurcation at around $t / T=15$. Both are increasing as the bifurcation is approached due to the increase in $\tau$. Phase lag is calculated from the difference between the times of the maxima in the forcing and the system response in each cycle. Response amplitude is calculated by taking half the difference between the maximal and minimal values in the system response in each cycle. Also plotted are the ratios of the second and third harmonic amplitudes to the amplitude of the fundamental harmonic with time using a sliding window of length 5 complete cycles against the time at the end of the sliding window. The window needs to be long enough to resolve the harmonics in the spectrum but short enough to keep $D_{m}$ approximately constant. For this example, where the harmonic amplitudes (and the nonlinearity of the response) are quite small, 5 cycles is the minimum to resolve the peaks. The sliding window is then advanced one cycle in the time series and the harmonic amplitudes are calculated for this new window. This process is iterated until the local bifurcation is reached to produce the lower panel in Fig. 3 which shows both harmonic amplitudes increasing.

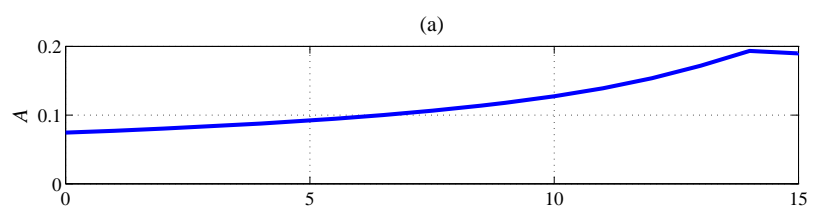

(b)

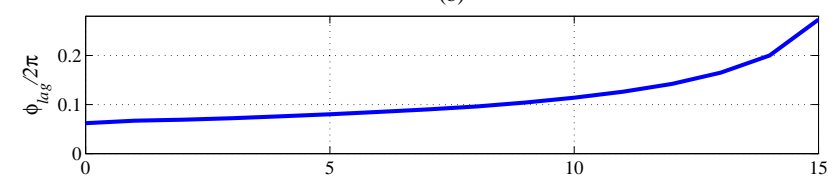

(c)

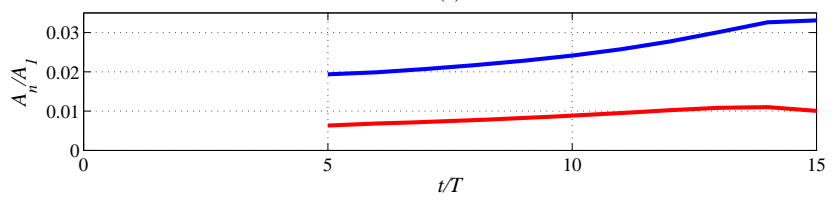

Figure 3. The early warning indicators, response amplification (upper panel), $A=\frac{D_{a} \tau}{\sqrt{1+\omega^{2} \tau^{2}}}$, and phase lag (middle panel), $\frac{\phi_{\mathrm{lag}}}{2 \pi}=$ $\frac{1}{2 \pi} \arctan (\omega \tau)$ calculated for the time series in Fig. 2. We have plotted these indicators prior to the bifurcation at $t / T \approx 15$. The 2 nd (blue) and 3rd (red) harmonic amplitudes $A_{n} / A_{1}$ are also plotted in the lower panel using a sliding window of 5 complete cycles. All indicators are increasing as expected.

We also plot the complete spectrum of the ratios $A_{n} / A_{1}$ against $T_{n} / T$ derived from a Fourier transform of the system response in Fig. 4. In the upper panel all parameters are the same as Fig. 2 except we have fixed $D_{m}$ in each of the two runs. In the first run $D_{m}=-2$, this is far from the bifurcation and one expects the system to behave more linearly (blue line). One sees a second harmonic around 2 orders of magnitude smaller than the linear response. In the second run $D_{m}=0.25$ and the orbit is much closer to the bifurcation (red line). The second harmonic has increased to about an order of magnitude smaller than the fundamental harmonic and a third harmonic is now also visible indicating the system has become more nonlinear.

To give an example of a climate system operating in this regime consider the annual variation in sea temperatures in Northern Hemisphere temperate regions. A rough estimate of the ocean surface mixed layer timescale gives $\tau \sim 10$ months and this surface layer is heated by the annual cycle of solar insolation to varying degrees throughout the year. Calculation of the phase lag for this $\tau$ and $T$ yields a lag of about 2.6 months, i.e. roughly the maximal and minimal sea temperatures are in September and March. Arctic sea ice extent also falls into this regime and we analyse this system in Sect. 4.

\subsubsection{A note about return maps}

Towards the upper range of $\omega \tau \sim 1$, specifically $\omega \tau>2 \pi$, return map analysis via statistical fixed point indicators becomes useful with the added caveat that the time series of 

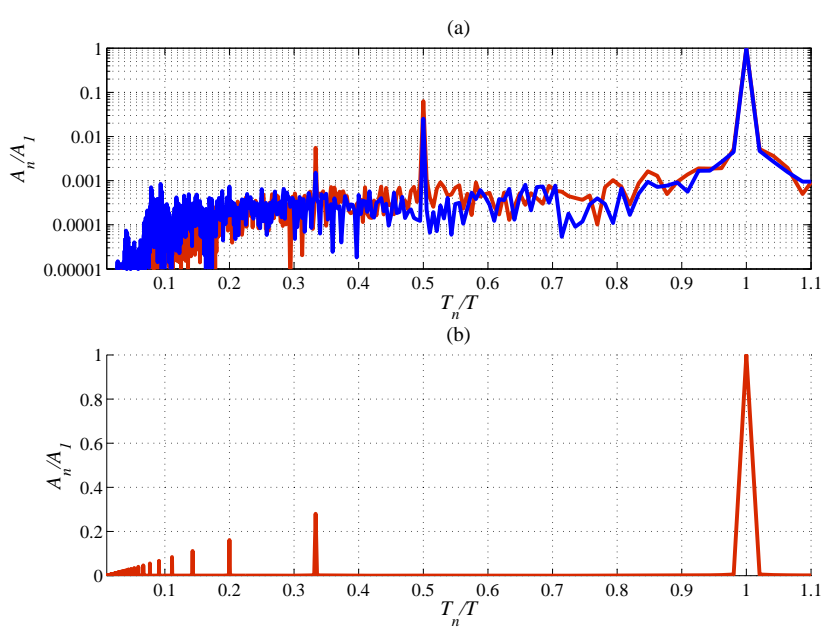

Figure 4. Ratio of the $n$th order harmonic amplitude to the fundamental harmonic amplitude $A_{n} / A_{1}$ against the ratio of the $n$th harmonic period to the fundamental harmonic period $T_{n} / T$. The dynamics of the system are described by equation 11 . In the upper panel parameters are fixed to $D_{a}=1 / 2, T=\pi$ (the same order as the system timescale $\tau$ ). The blue line is for $D_{m}=-2$ (far away from the bifurcation), the nonlinear response is dominated by the second harmonic at $T_{n} / T=1 / 2$ although small, about two orders of magnitude less than the linear response. The red line is $D_{m}=1 / 4$, close to the bifurcation the system response has become more nonlinear. The second harmonic $\left(T_{n} / T=1 / 2\right)$ is now almost 1 order of magnitude less and the third order harmonic ( $\left.T_{n} / T=1 / 3\right)$ is also prominent. In the bottom panel, we show the spectrum when the dynamics are very nonlinear. Parameters are set to $D_{m}=0, D_{a}=1 / 2, T=100 \pi$ so $\omega \tau \approx 1 / 100$. This is the slow forcing limit shown in Fig. 1 (red line) which has a very nonlinear relaxation oscillation type response. Note only odd harmonics ( $T_{n} / T=1 / 3,1 / 5,1 / 7, \ldots$ etc.) are present due to the system experiencing a symmetric potential requiring the solution, $x(t)$, to also have this symmetry.

the system must have enough cycles to produce statistically significant results. Return map analysis is complementary to phase lag and response amplification since these quantities start to asymptote when $\omega \tau>2 \pi$. This complementarity is illustrated in the following figures. The blue line in Fig. 5 is essentially the same as Fig. $2(\omega \tau \sim 1)$ except $D_{m}$ is varied over 100 cycles instead of 25 . This is because extra data points are needed to calculate the lag 1 autocorrelation of the return maps with any reliability. We have also added Gaussian white noise to Eq. (11) of standard deviation 0.01 as the return map method needs small perturbations with which to infer return times to the cycle. In Fig. 6 we have plotted the early warning indicators for this time series including the return map calculated with a sliding window of 25 cycles. The black lines are the theoretical curves and the blue lines are the estimated curves. The key point is the theory and estimated autocorrelations do not show anything in this regime $(\omega \tau \sim 1)$ however the phase lag and response amplification are clearly increasing.
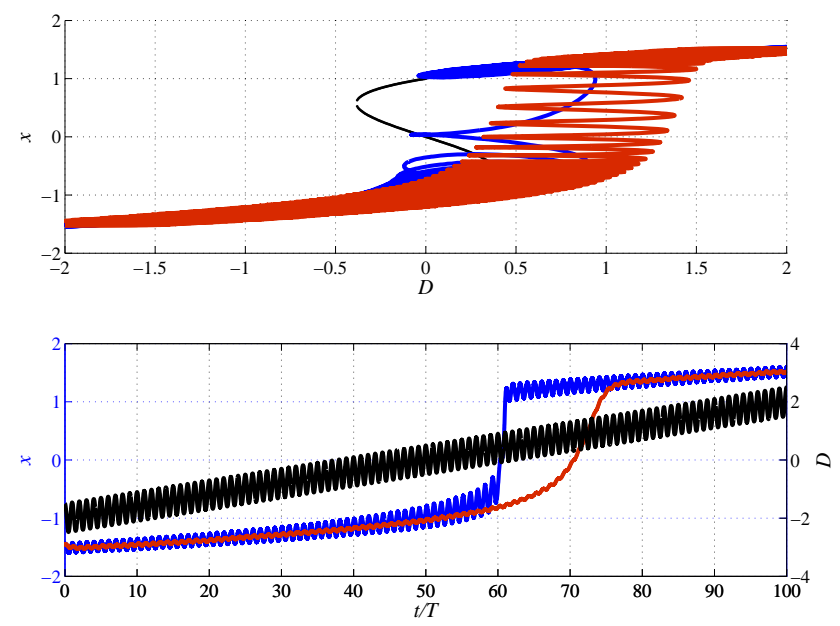

Figure 5. Same figure as Fig. 2 in the manuscript except the variation of $D_{m}$ is over more cycles to generate more points for a reliable return map analysis. Weak Gaussian white noise of standard deviation 0.01 is added to the system. Parameters are set to $D_{a}=1 / 2$ and $D_{m}$ is varied linearly with time between -2 and 2 over about 100 cycles. In the upper panel the black lines are the nullclines while the system response is the blue line for $T=\pi$ giving $\omega \tau \sim 1$ whereas the red line has a shorter period of $T=1 / 4$ to give $\omega \tau \sim 4 \pi$. These are plotted against $D(t)$. In the lower panel we have plotted these system responses as time series against the forcing (black line).

Conversely, the red lines in Figs. 5 and 7 are the same quantities but with a decreased period of forcing $(T=1 / 4$ so $\omega \tau \sim 4 \pi)$. This is a regime in which phase lag and response amplitude start to asymptote and are therefore not so useful to infer changing system timescale. However, lag 1 autocorrelation of the return map now becomes useful as can be seen in Fig. 7.

\subsection{Period of forcing much slower than system timescale, $\omega \tau \ll 1$}

When Eq. (11) is operating in this regime (period of forcing much greater than system timescale $T \gg \tau$ ) the system can adjust to changing $D(t)$ relatively quickly and effectively remains at a fixed point. $D(t)$ can therefore be modelled as a slow constant, control parameter and all the usual timescale separation assumptions apply. Fixed point indicators such as lag 1 autocorrelation are then good early warning indicators of local bifurcations. In contrast, phase lag and response amplitude are not useful as these quantities asymptote to $\phi_{\text {lag }} \rightarrow 0$ and $\rightarrow \tau$ respectively. The system state $x$ is plotted against $D$ and against $t$ as the red line in Fig. 1.

An example of a system that has the correct timescale separation and periodic forcing are the glacial and/or interglacial cycles that have the slow build, fast collapse type behaviour of relaxation oscillations. Ice sheets have timescales in the order of thousands of years forced by the solar insolation variation of Milankovitch cycles. The forcing is a superpo- 

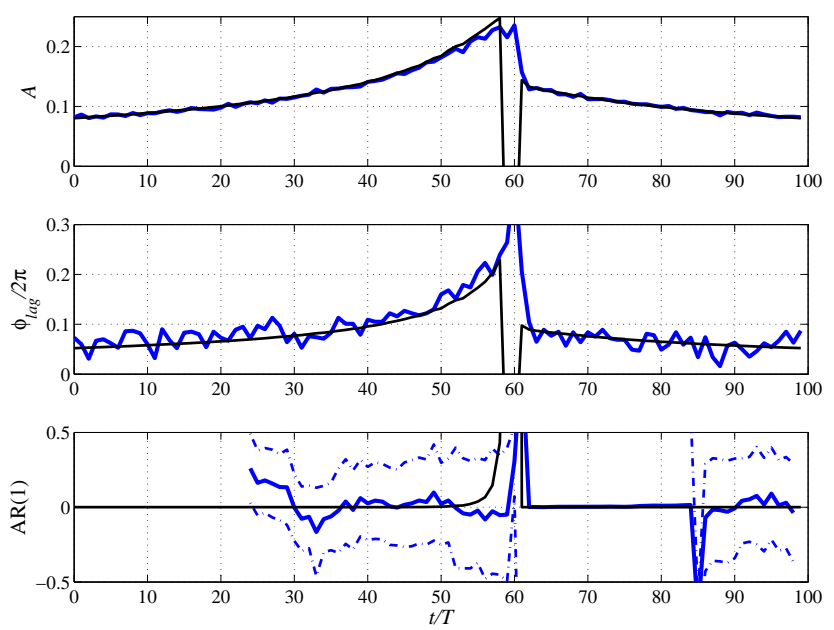

Figure 6. $\omega \tau \sim 1$ : The early warning indicators, response amplification (upper panel), $A=\frac{D_{a} \tau}{\sqrt{1+\omega^{2} \tau^{2}}}$, and phase lag (middle panel), $\frac{\phi_{\text {lag }}}{2 \pi}=\frac{1}{2 \pi} \arctan (\omega \tau)$ calculated for the blue time series in Fig. 5. In the lower panel, lag 1 autocorrelation of a sliding window of 25 points of the return map is plotted with standard errors (dashed lines) on the estimate. Black lines are theoretical curves of all the quantities. The key point is that phase lag and amplitude response are useful quantities in this regime however the return map is not.

sition of many different sinusoidal frequencies, the dominant ones having periods of $41 \mathrm{kyr}$ (related to the obliquity of Earth's orbit), 19 and $23 \mathrm{kyr}$ (related to the precession). Current thinking, however, favours more complex, two- and higher-dimensional dynamics to model these cycles than the single variable models we consider in this paper (Saltzman, 2002; Crucifix, 2012; Saedeleer et al., 2013; Crucifix, 2013).

The spectrum of a very nonlinear, relaxation oscillation type, dynamics is illustrated in the lower panel of Fig. 4. This is the spectrum of the slow forcing run (red line) in Fig. 1. Only odd harmonics appear in its spectrum because the static potential $V=-\int \dot{x} \mathrm{~d} x$ is symmetric about $x$ for this value of $D_{m}=0$, i.e. $V(x)=V(-x)$ and therefore any solution of $\dot{x}$ must also have this symmetry, $x(t+T / 2)=-x(t)$. Only odd harmonics have this property. ${ }^{2}$

\footnotetext{
${ }^{2}$ This is not sufficient though as there are other parameter settings that feature the second harmonic and also have the same symmetric potential, i.e. $D_{m}=0$ and $T=\pi$ in Fig. 1 (blue line). The difference is that the runs featuring second harmonic responses only experience a limited part of the potential, not the full symmetric potential. Even though the potential is the same, the forcing is quick enough to trap the system in an orbit in just one of the two potential wells. This local potential well is asymmetric and what the system sees is effectively described by a Taylor expansion around the centre of that well. In contrast the relaxation oscillation type run travels across both wells equally and therefore sees the global symmetric potential requiring an odd harmonic solution. This is not a generic case however.
}
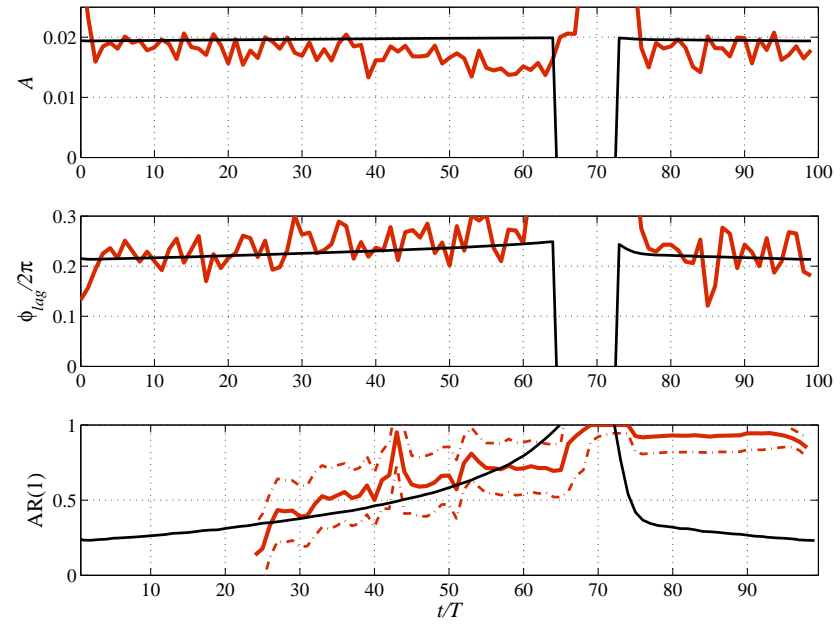

Figure 7. $\omega \tau \sim 4 \pi$ : The early warning indicators, response amplification (upper panel), $A=\frac{D_{a} \tau}{\sqrt{1+\omega^{2} \tau^{2}}}$, and phase lag (middle panel), $\frac{\phi_{\mathrm{lag}}}{2 \pi}=\frac{1}{2 \pi} \arctan (\omega \tau)$ calculated for the red time series in Fig. 5. In the lower panel, lag 1 autocorrelation of a sliding window of 25 points of the return map is plotted with standard errors (dashed lines) on the estimate. Black lines are theoretical curves of all the quantities. Phase lag and amplitude response have now asymptoted and are not useful quantities however the return map now becomes useful.

\subsection{Period of forcing much faster than system timescale, $\omega \tau \gg 1$}

The system state $x$ is plotted against $D$ and against $t$ as the green line in Fig. 1. The forcing is changing much faster than the system can respond so the system effectively looks static and all the dynamics come from the forcing directly. In this case we can place $D(t)$ in the fast dynamics. However, not all of the other assumptions for use of lag 1 autocorrelation in a fixed point analysis are satisfied. It is true that $D(t)$ is independent of $x$, however it is not uncorrelated with itself at different times and therefore cannot strictly be modelled as a normally distributed random variable, although at first glance it looks as though it is again possible to use usual fixed point early warning techniques so one must be careful. In this regime, phase lag and response amplification asymptote and again are not very useful to detect a trend in increasing timescale. Phase lag, $\phi_{\operatorname{lag}} \rightarrow \pi / 2$ and response amplification $\rightarrow \frac{1}{\omega}$ so one may only infer $\tau \gg T$.

An example of a system approximately modelled by this limit is the global terrestrial vegetation carbon which has a dominant timescale on the order of decades, much larger than its periodic forcing, the annual cycle of solar insolation. This dominant timescale comes from the large long term carbon storage, e.g. the timescale taken for a forest to regrow once cut down. One sees this phase lag of quarter of a cycle in 


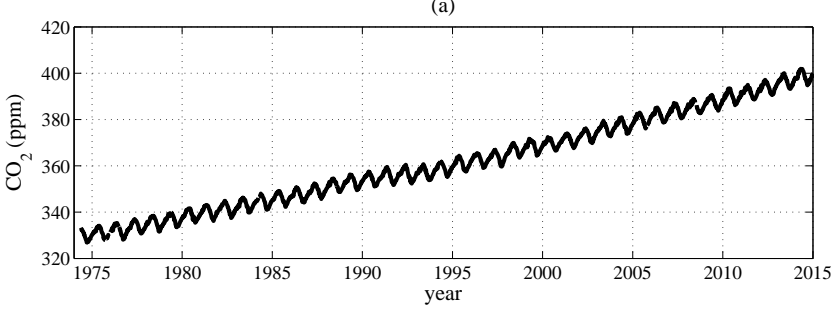

(b)

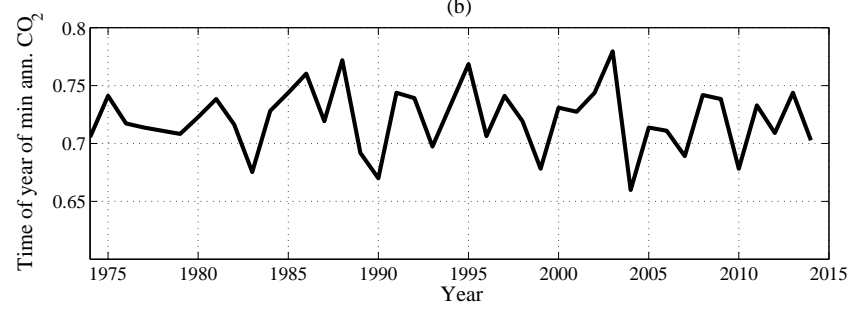

Figure 8. Atmospheric $\mathrm{CO}_{2}$ concentration recorded at Mauna Loa against time in the upper panel. In the lower panel we have plotted the minimum annual $\mathrm{CO}_{2}$ concentration against year. One notices the minimum $\mathrm{CO}_{2}$ concentration occurs roughly $3 / 4$ of the way through the year. This is because maximal carbon uptake occurs during the Northern Hemisphere summer from the terrestrial vegetation and it is maximally lagged behind the maximum in the Northern hemisphere solar insolation (best growing conditions) by $1 / 4$ of a cycle because of the timescale difference between the response of the system and the period of the forcing.

the annual minimum of the Mauna $\mathrm{Loa} \mathrm{CO}_{2}$ record ${ }^{3}$ relative to the Northern Hemisphere solar insolation maximum. This lagged annual minimum in the integrated response of the total atmospheric carbon results from the dominance of the Northern Hemisphere's mid latitude terrestrial vegetation carbon in the global carbon flux. We have plotted the Mauna Loa $\mathrm{CO}_{2}$ record and the time of year of the minimum concentration in Fig. 8.

\section{Looking for a tipping point in Arctic sea ice satellite observations}

There has been much research on a possible local bifurcation and tipping point in the Arctic sea ice, see for example Armour et al. (2011), Eisenman and Wettlaufer (2009), Lindsay and Zhang (2005), Livina and Lenton (2013), Ridley et al. (2012) and Wang and Overland (2012). This possible bifurcation in the sea ice cover may be due to the well-known ice albedo feedback first studied by Budyko (1969) and Sellers (1969). When ice is present it reflects a high proportion of the incoming solar radiation due to its higher albedo yet when it starts receding the darker ocean absorbs more radiation increasing heating and promoting more sea ice retreat.

\footnotetext{
${ }^{3}$ Pieter Tans, NOAA/ESRL (www.esrl.noaa.gov/gmd/ccgg/ trends/) and Ralph Keeling, Scripps Institution of Oceanography (www.scrippsco2.ucsd.edu/)
}

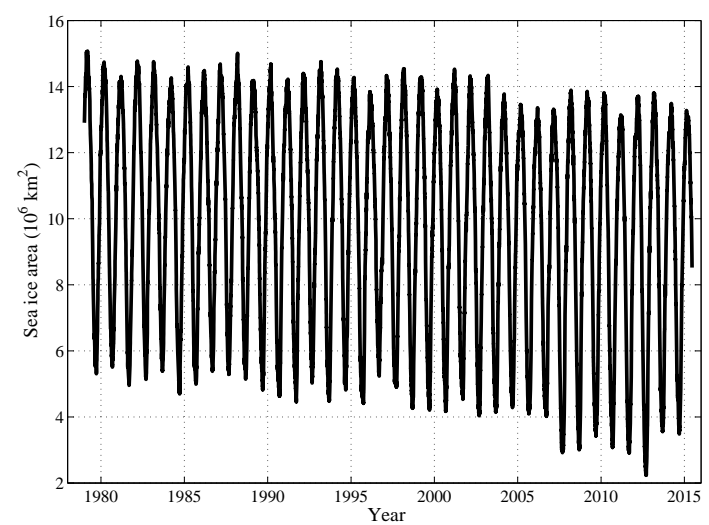

Figure 9. Arctic sea ice area satellite observations from 1979 to present day (2015) obtained from The Cryosphere Today project of the University of Illinois.

This feedback can result in instability and multiple steady states.

We calculate all the previously mentioned early warning indicators for a time series of Arctic sea ice area satellite observations from 1979 to present day. That is we calculate phase lag, response amplitude, relative size of the 2 nd and 3rd harmonics and the lag 1 autocorrelation of the return map with time to look for signs of critical slowing down that might indicate the approach of a local bifurcation or "tipping point" in the Arctic sea-ice. We also calculate the complete Fourier spectra for the entire time series as a linearity check. In Fig. 9 satellite observations of Arctic sea ice area are plotted against year. Sea ice area data were obtained from The Cryosphere Today project of the University of Illinois. This data set ${ }^{4}$ uses SSM/I and SMMR series satellite products and spans 1979 to present at daily resolution.

In Fig. 10 we plot the amplitude of the sea ice area annual cycle and the phase lag between the sea ice area minimum and maximum during each cycle. We assume the maximal and minimal driving occurs at the same time as maximal and minimal of the solar insolation, that is, the midpoint and end point of the year respectively to obtain phase lags. To limit the impact of high-frequency variability on the location of the extrema, we have smoothed the daily data with a sliding window of 30 days.

From Fig. 10 we see the cycle amplitude is increasing with time although the phase lag does not appreciably change. We first make some rough calculations to see if these plots are consistent with each other: from the phase lag figure, a timescale of $\tau \sim[0.33,0.5]$ years from the lag of $[0.18,0.2]$ of a cycle can be inferred. If we assume for the moment the amplitude of the forcing $D_{a}$ is not changing throughout the time period of the observations (this may not be true) and take the smallest value in the range for

\footnotetext{
${ }^{4}$ http://arctic.atmos.uiuc.edu/cryosphere/timeseries.anom. 1979-2008
} 

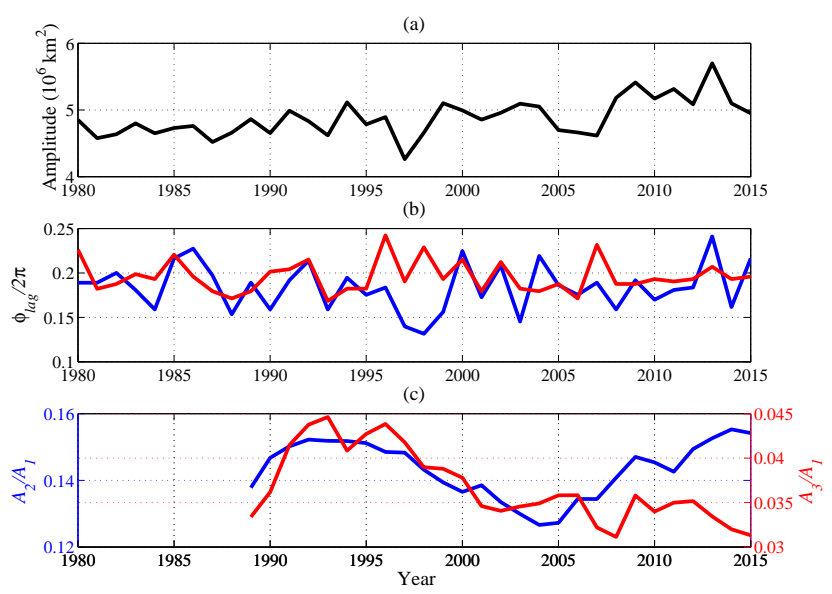

Figure 10. In the upper panel amplitude of sea ice area within each cycle is plotted against year. In the middle panel phase lag is plotted between the sea ice area minimum (red line) and maximum (blue line) and the solar insolation minimum and maximum respectively against the year. In the lower panel, the 2nd (blue) and 3rd (red) harmonic amplitudes $A_{n} / A_{1}$ are plotted against year end using a sliding window of 10 years. The amplitude is increasing however the phase lag is not. Harmonic amplitudes also show no convincing trend.

$\tau_{1978}=0.33$ years occurring in 1978 and the largest value in $2015, \tau_{2015}=0.5$ years we can make a rough calculation of how much the sea ice amplitude would have increased, i.e. $\frac{A_{2015}}{A_{1978}}=\frac{\tau_{2015}}{\tau_{1978}} \sqrt{\frac{1+\omega^{2} \tau_{1978}^{2}}{1+\omega^{2} \tau_{2015}}} \approx 1$ 1.06. From Fig. 10 we take the amplitude at 1978 to be $A_{1978} \sim 4.5$ and at 2015 to be $A_{2015} \sim 5$ we find $\frac{A_{2015}}{A_{1978}}=1.11$. These values could therefore be consistent with a constant $D_{a}$ and a changing timescale. However, the timescales inferred from either the phase lag or amplitude are not changing appreciably and therefore it seems unlikely the system is approaching a local bifurcation.

We note that the phase lag is a more robust indicator. This is because the phase lag depends only on the product of the frequency of the forcing and the system timescale whereas the amplitude depends additionally on the amplitude of the driving, $D_{a}$, which may well be changing throughout the observational period and could account for some or all of the increase seen in the amplitude in Fig. 10. Although the solar insolation will be a large component of the forcing amplitude and is essentially fixed, other factors such as clouds as well as air and sea temperatures will also factor into the driving amplitude. Geometrical constraints imposed by land masses affecting the maximal extent of the sea ice will also influence the amplitude of the sea ice oscillation when ice extent is large (Eisenman, 2010). In contrast, we can take the frequency of the driving to be essentially fixed by the annual solar insolation cycle making the phase lag more robust.

In the lower panel of Fig. 10 we have plotted the ratio of the second and third harmonic amplitudes to the amplitude of the fundamental harmonic with time using a sliding win-

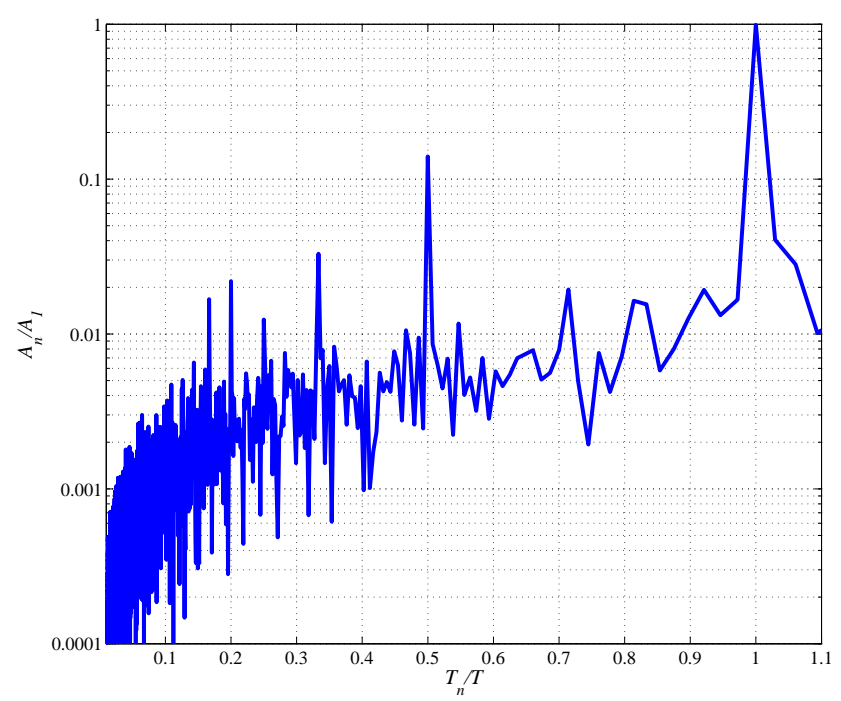

Figure 11. Ratio of the $n$th order harmonic amplitude to the fundamental harmonic amplitude $A_{n} / A_{1}$ found from the Fourier transform of the Arctic sea ice area time series against the ratio of the $n$th harmonic period to the fundamental harmonic period $T_{n} / T$. One can see the Arctic sea ice response features prominent second, third, forth, fifth and sixth harmonics in its spectrum.

dow of 10 complete cycles against the year at the end of the window. We have used the minimal window length needed to resolve both harmonics reliably. This indicator also shows no clear trend with time.

We have also calculated the lag 1 autocorrelation of the return map. From phase lag, the estimate timescale of the sea ice is 0.5 years $(\omega \tau \sim \pi)$ which is less than $\tau / T>1$ needed for a reliable estimate. However, the estimated timescale is uncertain and it is conceivable the return map analysis might work. As there are only 37 complete years of data, any return map time series has a maximum of 37 data points. To discern any trend in the autocorrelation one needs as many windows as possible, however this results in a decreasing number of data points per time series and an increasing error in the estimate. We have therefore chosen a sliding window of $20 \mathrm{cy}-$ cles although the results are invariant to this choice, always being very uncertain. We linearly detrend the cycle in each sliding window and then create the return map time series from that detrended window. One can also choose at which point in the cycle one wants to take the return map from and this additional freedom is utilised in the right hand panel in Fig. 12. Lag 1 autocorrelation is plotted against sliding window end year ( $x$ axis) and day of the year in each cycle the return map generated on (the $y$ axis). We create new return maps every 10 days giving 36 different points within each cycle. As seen in the figure, autocorrelation depends very heavily on where in the cycle one chooses to generate the map, a sign that the return map is not a good approach for this system. A good return map should be largely invariant to where in the cycle it is taken provided the cycle is stable and 

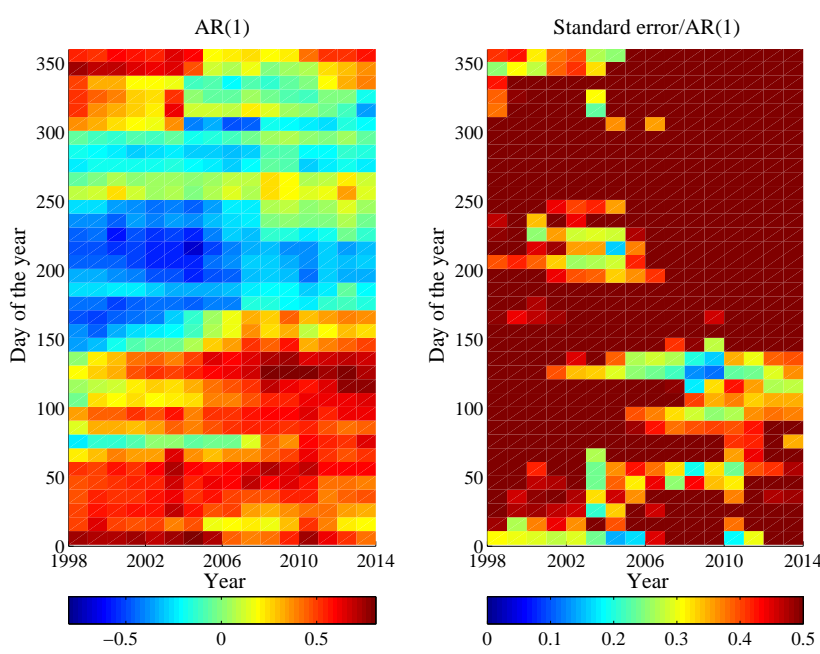

Figure 12. Left panel: Lag 1 autocorrelation of the return map against sliding window end year using a sliding window of 20 years ( $x$ axis) and point within the cycle the return map is created on the $y$ axis (we create return maps every 10 days). One sees that autocorrelation depends very heavily on where in the cycle one chooses to generate the return map. Right panel: standard error of the autocorrelation return map estimate divided by the estimate against sliding window end year. The estimate is very uncertain almost everywhere with standard errors generally being at least half as big as the estimate.

not changing. In the left hand panel of Fig. 12 we plot the standard error divided by the autocorrelation. Note that most estimates of lag 1 autocorrelation have standard errors larger than half their value giving very uncertain estimates. In an effort to reduce the uncertainty in the estimate we have also taken the mean autocorrelation over all points in the cycle the return map is taken in Fig. 13. The mean lag 1 autocorrelation is $0.16 \pm 0.26$ which corresponds to a (very uncertain) timescale of $\tau \approx 0.55$ years. This is consistent with the estimates from the phase lag. This also suggests that the sampling interval $T>\tau$ and therefore determining the timescale using the return map approach is difficult. We have increased the sliding window to 37 years to minimize the standard error in the estimate, however one will not be able to then see a trend in autocorrelation. Even so, the standard errors are still greater than half the estimate.

We have also plotted the full spectrum of the ratios $A_{n} / A_{1}$ for the entire time series in Fig. 11. We note the nonlinear effects are quite prominent in this system, second and third harmonics are around an order of magnitude smaller than the linear response, although we can still probably get away with the linear analysis. Forth, fifth and sixth harmonics are also visible. These nonlinearities may be due to albedo effect or to the geometrical effects of the Arctic ocean basin (Eisenman, 2010).

To conclude, from this simple analysis it seems that the system's timescale and therefore stability is not changing

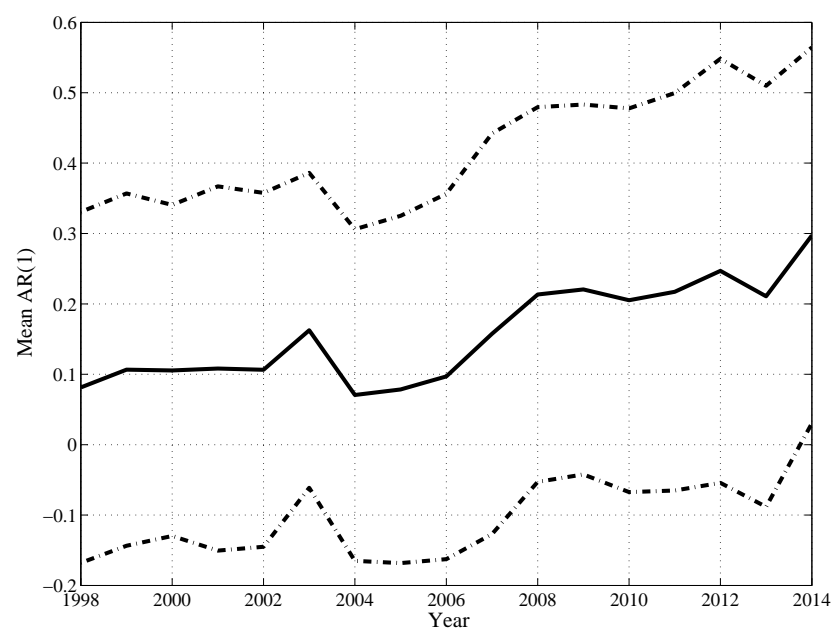

Figure 13. Mean lag 1 autocorrelation of the return map across all starting points within the cycle using a sliding window of 20 years. This is the same as Fig. 12 with the mean taken along the $y$ axis. Estimated autocorrelation is still very uncertain. The mean is the solid line with the dotted lines being the mean plus or minus the standard error. The mean value across all years $0.16 \pm 0.26$ which corresponds to a (very uncertain) timescale of $\tau \approx 0.55$ years.

appreciably if at all and it is unlikely to be approaching a local bifurcation. However, simple theoretical models, such as Eisenman and Wettlaufer (2009), Eisenman (2012) and Bathiany et al. (2016, who also used a return map approach) suggest that the sea ice timescale does not change very much approaching the bifurcation, even decreasing slightly before rapidly changing over a very small interval and therefore would be very hard to detect if present.

\section{Conclusions}

Much previous work on detecting local bifurcations from time series required one to be able to partition the universe into widely separated timescales and model the system dynamics as overdamped. When this is the case one can use the usual, statistical fixed point early warning indicators of increasing lag 1 autocorrelation and variance since these indicators measure the system's response to small perturbations away from its fixed point by the fast, noisy processes. It is the response to this small, noisy forcing that allows one to measure the system's timescale. The systems we have been looking at in this paper do not have fast or random forcing. The systems considered here have deterministic forcing with a period roughly that of its timescale although the dynamics are still overdamped. Deterministic forcing again allows one to infer the system's timescale simply by measuring the response to the forcing without the need for large amounts of data required by statistical quantities for robust estimates. We used two analogous early warning indicators to lag 1 autocorrelation and variance in these systems; these were phase lag 
and response amplification respectively. Just as autocorrelation is more robust as an indicator (it is a function of fewer parameters), the same is true of phase lag, only depending on the frequency of the forcing and the timescale of the system. The system response amplification also depends on the amplitude of forcing, which in many circumstances is probably difficult to measure.

We also used a Fourier transform of the time series to quantify how nonlinear the system is behaving and whether the linear approximations usually made are good. Further, by using a sliding window within the time series, one may also look at the evolution of the harmonic amplitudes as a further early warning indicator.

We also discussed return map methods that essentially convert a periodic attractor to a fixed point type so that one may use the usual fixed point indicators. We also showed there was a complementarity between return map indicators and phase lag and response amplification, the latter being more useful for regimes in which $\omega \tau \sim 1$ and the former being more useful when $\omega \tau>2 \pi$.
We applied these indicators to satellite observations of Arctic sea ice area, a system whose period of forcing, effectively the annual cycle of insolation, is similar to the timescale of the system. This is also a system that has been conjectured to have a tipping point due to a local bifurcation. We did not find any detectable critical slowing down and therefore signs of this bifurcation. It should be noted, however, that simple models of the sea ice suggest critical slowing down only occurs very close to the bifurcation, making it very hard to detect.

\section{Data availability}

The data sets of the Mauna Loa $\mathrm{CO}_{2}$ record (Tans and Keeling, 2015) and the Arctic sea-ice area (Chapman et al., 2015) analyzed in this manuscript are publicly available at www. esrl.noaa.gov/gmd/ccgg/trends/ and http://arctic.atmos.uiuc. edu/cryosphere/timeseries.anom.1979-2008 respectively. 
Appendix A: Phase lag and response amplification with arbitrary periodic forcing

Phase lag and response amplification can be found for the more general case of any type of periodic forcing $D$ by solving

$\dot{x}+\frac{x}{\tau}=D(t)$

$\tau$ the timescale of the system (the $e$ folding time). For any periodic forcing, $D(t)$ with period $T$ can be written as the Fourier series

$D(t)=\sum_{i=0}^{N} B_{i} \cos \left(\omega_{i} t+\chi_{i}\right)$.

$B_{i}$ are the amplitudes of the different component sinusoidal waves, $\omega_{i}=\frac{2 \pi i}{T}$ are the frequencies of the components and $\chi_{i}$ are the phases of each of the components. As the equation is linear the superposition principle holds. That is, we assume the solution has the form

$x(t)=\sum_{i=0}^{N} x_{i}(t)$

by setting all but the $i$ th term of the driving to zero we can solve the $N+1$ equations

$\dot{x}_{i}+\frac{x_{i}}{\tau}=B_{i} \cos \left(\omega_{i} t+\chi_{i}\right)$

for each $x_{i}(t)$. These solutions can be superposed to obtain the full solution to any periodic driving term.
This is

$$
\begin{aligned}
x(t) & =\sum_{i=0}^{N} \frac{\tau B_{i}}{\sqrt{1+\omega_{i}^{2} \tau^{2}}}\left[\cos \left(\omega_{i} t+\chi_{i}-\arctan \left(\omega_{i} \tau\right)\right)\right. \\
& \left.-e^{-\frac{t}{\tau}} \cos \left(\chi_{i}-\arctan \left(\omega_{i} \tau\right)\right)\right]+x_{0} e^{-\frac{t}{\tau}}
\end{aligned}
$$

which settles into orbit

$x(t)=\sum_{i=0}^{N} \frac{\tau B_{i}}{\sqrt{1+\omega_{i}^{2} \tau^{2}}} \cos \left(\omega_{i} t+\chi_{i}-\arctan \left(\omega_{i} \tau\right)\right)$

when $t \gg \tau$, that is, the solution is just the sum of each of the forcing components $i$, each with a response amplification of

$\frac{\tau}{\sqrt{1+\omega_{i}^{2} \tau^{2}}}$

and a response lagging the forcing with a phase of

$\phi_{i}^{\text {lag }}=\arctan \left(\omega_{i} \tau\right)$.

One can find out what these phase lags and amplitudes are by taking the Fourier transform of the time series of both the forcing and response. 
Acknowledgements. The research leading to these results has received funding from the European Union Seventh Framework Programme FP7/2007-2013 under grant agreement no. 603864 (HELIX). We are grateful to Peter Ashwin, Peter Cox, Michel Crucifix, Vasilis Dakos, Henk Dijkstra, Jan Sieber, Marten Scheffer and Appy Sluijs for the fruitful discussions over beers and balls.

Edited by: J. Annan

\section{References}

Armour, K. C., Eisenman, I., Blanchard-Wrigglesworth, E., McCusker, K. E., and Bitz, C. M.: The reversibility of sea ice loss in a state-of-the-art climate model, Geophys. Res. Lett., 38, L16705, doi:10.1029/2011GL048739, 2011.

Bathiany, S., van der Bolt, B., Williamson, M. S., Lenton, T. M., Scheffer, M., van Nes, E., and Notz, D.: Trends in sea-ice variability on the way to an ice-free Arctic, The Cryosphere Discuss., doi:10.5194/tc-2015-209, in review, 2016.

Budyko, M. I.: The effect of solar radiation variations on the climate of the Earth, Tellus, 21, 611-619, 1969.

Carpenter, S., Cole, J., Pace, M. L., Batt, R., Brock, W. A., Cline, T., Coloso, J., Hodgson, J. R., Kitchell, J. F., Seekell, D. A., Smith, L., and Weidel, B.: Early Warnings of Regime Shifts: A WholeEcosystem Experiment, Science, 332, 1079-1082, 2011.

Chapman, W. and National Center for Atmospheric Research Staff (Eds.): The Climate Data Guide: Walsh and Chapman Northern Hemisphere Sea Ice, retrieved from https://climatedataguide.ucar.edu/climate-data/ walsh-and-chapman-northern-hemisphere-sea-ice, available at: http://arctic.atmos.uiuc.edu/cryosphere/timeseries.anom. 1979-2008, 2015.

Crucifix, M.: Oscillators and relaxation phenomena in Pleistocene climate theory, Philos. T. R. Soc. A, 370, 1140-1165, 2012.

Crucifix, M.: Why could ice ages be unpredictable?, Clim. Past, 9, 2253-2267, doi:10.5194/cp-9-2253-2013, 2013.

Dakos, V., Scheffer, M., van Nes, E. H., Brovkin, V., Petoukhov, V., and Held, H.: Slowing down as an early warning signal for abrupt climate change, P. Natl. Acad. Sci. USA, 105, 1430814312, 2008.

Domb, C., Green, M. S., and Lebowitz, J. (Eds.): Phase transitions and critical phenomena, vol. 1-20, Academic Press, 1972-2001.

Eisenman, I.: Geographic muting of changes in the Arctic sea ice cover, Geophys. Res. Lett., 37, L16501, doi:10.1029/2010GL043741, 2010.

Eisenman, I.: Factors controlling the bifurcation structure of sea ice retreat, J. Geophys. Res., 117, D01111, doi:10.1029/2011JD016164, 2012.

Eisenman, I. and Wettlaufer, J. S.: Nonlinear threshold behaviour during the loss of Artic sea ice, P. Natl. Acad. Sci. USA, 106, 28-32, 2009.

Gammaitoni, L., Hänggi, P., Jung, P., and Marchesoni, F.: Stochastic resonance, Rev. Mod. Phys., 70, 223-287, 1998.

Held, H. and Kleinen, T.: Detection of climate system bifurcations by degenerate fingerprinting, Geophys. Res. Lett., 31, L23207, doi:10.1029/2004GL020972, 2004.

Jung, P. and Hänggi, P.: Hopping and phase shifts in noisy periodically driven bistable systems, Z. Phys. B Con. Mat., 90, 255-260, 1993.
Kuznetsov, Y. A.: Elements of Applied Bifurcation Theory, third edition, Springer, 2004.

Lenton, T. M.: Early warning of climate tipping points, Nature Climate Change, 1, 201-209, 2011.

Lindsay, R. W. and Zhang, J.: The thinning of the Arctic sea ice, 1988-2003: Have we passed a tipping point?, J. Clim., 18, 48794894, 2005.

Livina, V. N. and Lenton, T. M.: A recent tipping point in the Arctic sea-ice cover: abrupt and persistent increase in the seasonal cycle since 2007, The Cryosphere, 7, 275-286, doi:10.5194/tc-7-2752013, 2013.

Main, I. G.: Vibrations and Waves, Cambridge University Press, 3rd Edn., 1993.

McNamara, B. and Wiesenfeld, K.: Theory of stochastic resonance, Phys. Rev. A, 39, 4854-4869, 1989.

Ridley, J. K., Lowe, J. A., and Hewitt, H. T.: How reversible is sea ice loss?, The Cryosphere, 6, 193-198, doi:10.5194/tc-6-1932012, 2012.

Saedeleer, B. D., Crucifix, M., and Wieczorek, S.: Is the astronomical forcing a reliable and unique pacemaker for climate? A conceptual model study, Clim. Dyn., 40, 273-294, 2013.

Saltzman, B.: Dynamical Paleoclimatology: Generalized theory of global climate change, Academic Press, 2002.

Scheffer, M., Carpenter, S. R., Lenton, T. M., Bascompte, J., Brock, W., Dakos, V., van de Koppel, J., van de Leemput, I. A., Levin, S. A., van Nes, E. H., Pascual, M., and Vandermeer, J.: Anticipating Critical Transitions, Science, 338, 344-348, 2012.

Sellers, W. D.: A global climate model based on the energy balance of the Earth-atmosphere system, J. Appl. Meteorol., 8, 392-400, 1969.

Shneidman, V. A., Jung, P., and Hänggi, P.: Power spectrum of a driven bistable system, Europhys. Lett., 26, 571-576, 1994.

Strogatz, S. H.: Nonlinear Dynamics and Chaos, Westview Press, 2001.

Tans, P., and Keeling, R.: Mauna Loa $\mathrm{CO}_{2}$ weekly mean, available at: ftp://aftp.cmdl.noaa.gov/products/trends/co2/co2_ weekly_mlo.txt, 2015.

Thompson, J. M. T. and Sieber, J.: Predicting climate tipping as a noisy bifurcation: A review, Int. J. Bifurcat. Chaos, 21, 399-423, 2011.

Thompson, J. M. T. and Stewart, H. B.: Nonlinear Dynamics and Chaos, second edition, John Wiley and Sons, Ltd., 2002.

Wang, M. and Overland, J. E.: A sea ice free summer Arctic within 30 years: An update from CMIP5 models, Geophys. Res. Lett., 39, L18501, doi:10.1029/2012GL052868, 2012.

Wiesenfeld, K.: Noisy precursors of nonlinear instabilities, J. Stat. Phys., 38, 1071-1097, 1985.

Wiesenfeld, K. and McNamara, B.: Small-signal amplification in bifurcating dynamical systems, Phys. Rev. A, 33, 629-641, 1986.

Williamson, M. S. and Lenton, T. M.: Detection of bifurcations in noisy coupled systems from multiple time series, Chaos: An Interdisciplinary Journal of Nonlinear Science, 25, 036407, doi:10.1063/1.4908603, 2015. 